Chapter 20 on representations as sums of 2 or 4 rational integer squares provides an opportunity to sketch the arithmetic of quaternions, and serves as an introduction to Waring's problem for cubes and fourth powers in Chapter 21, where Tarry's problems, sums $\sum \pm x_{i}^{k}$, and equal sums of two $k$ th powers are also briefly considered. Any reader of this notice who is looking for something hard to do is invited to observe page 336, (f). The notes on pages 334-337 summarize the main results up to 1938 on Waring's problem.

Chapter 22, the third in the book on the series of primes, follows Landau's proofs of Tchebychef's theorems, and pushes the analysis a little further to obtain Mertens' approximation to $\prod_{p \leqq x}\left(1-p^{-1}\right), p$ prime. The chapter concludes with a note on "round" numbers and a theorem of the "almost all" type on the order of the number of divisors of $n$.

As the last of the authors' introductions, we mention Chapter 13 on certain Diophantine equations $\left(x^{n}+y^{n}=z^{n}, n=2,3,4\right)$, the expression of $m$ as a sum of rational cubes, $x^{3}+y^{3}=3 z^{3}$, and equal sums of two cubes.

The foregoing sample from the two dozen chapters covering 400 pages may give some idea of the extraordinary richness of the material, and suggest the justice of the authors' own characterization of their work as "a series of introductions" to a vast and many-sided theory. They have presented these introductions in a manner that should stimulate a reader to continue beyond some of them; and it seems safe to say a great deal more than what they themselves say, "we can hardly have failed completely, the subject-matter being so attractive that only extravagant incompetence could make it dull." The book is anything but dull; in fact it is as lively as the proverbial (not the English) cricket.

\title{
E. T. BELL
}

Le Concours de l'Analyse Mathêmatique a l'Analyse Expérimentale des Faits Statistiques. By Georges Hostelet. (Actualités Scientifiques et Industrielles, no. 585; Le Progrès de l'Esprit; Exposés publiés sous la direction de L. Brunschvicg.) Paris, Hermann, 1937. 70 pp.

The first section can be summed up by stating that the experimenter believes that the basic laws on which statistics are founded have been proven by the mathematician, while the mathematician believes that these laws have been demonstrated by the experimenter.

In the next section the author states that the mathematical analysis of statistical facts are contained in the intuitive-empirical notion of species, which implies notions of means, errors of means, frequencies and limits of these errors. He gives the conditions, which a statistical fact must satisfy in order that the arithmetic mean signifies the best value, defines the analytic and synthetic indicator of the degree of correlation, outlines the conditions necessary for linear correlation and considers that mathematical analyses are auxiliaries to experimental analyses of measurable facts when measurements present numerical relations expressible in explicit functions.

Section three contains postulates on which mathematical and experimental reasoning depend, answers to objections Fréchet made concerning probability and its use in the analysis of statistical facts, the necessary steps for verifying an hypothesis and an explanation of the difference between mathematical and experimental reasoning.

Chapter IV presents the three modes of scientific investigation, viz: the empiricalintuitive mode, the deductive-abstract mode and the abstract-experimental mode and points out their limitations. 
The following chapter contains discussions pertaining to philosophy, philosophic and scientific certitude, philosophic and scientific attitudes, types of judgments and modes of thought used in reasoning, and comparisons between social, physical and mathematical sciences. The author states that his efforts, by experimental analysis of observations of statistical facts, have been to disengage their characteristics and their correlations and to establish the limitative conditions of the experimental validity concerning them. In order to reveal the danger of the abstract reasoning, which menaces the course of mathematical analyses in the experimental analyses, he insists on the existence of the conditions which should be realized in order that the formulas, obtained from mathematics, be legitimate.

In the first chapter of Part II the author states the ideas of Reichenbach concerning causality, uncertainty of scientific propositions and predicting future events. He compares his views with those of Reichenbach, examines the notion of uncertainty in science, states that the idea connected by Reichenbach to the idea of uncertainty is associated with the absence of causality, shows how causality is connected with probability and discusses the relations of philosophy to scientific procedure of experimental analysis.

Chapter VII contains de Broglie's definition of determinism, an explanation of how de Broglie considers causality and determinism and his illustration relating to electrons shot from a cannon, bombarding a surface of a crystal, quotations from Planck, Einstein, Lord Rutherford relating to causality, a discussion of philosophic and scientific determinism in relation to causality and de Broglie's views on probability and frequency.

The following chapter presents the position of Barzin on notions of causality and determinism, which are characteristic of the attitude of logicians. Logic researches concerning probability establish the necessity of affirming a principle of induction in the sense that it adopts universal determinism; each theory of probability implies logically causal relations. By the use of perfect and imperfect games of chance the author compares his views with those of Barzin and points out the difference between the calculus of probability and statistical laws. Barzin believes that the principle of statistical induction rests on the affirmation of a determinism; Hostelet believes that this principle implies causality.

The last two chapters treat of comparisons of the philosophic and scientific attitude and the role of scientific methodology. Near the close of the book the author states that to practice the scientific method is to give the mind more security in the acquisition of knowledge, is to increase the surety and the power of its faculties of intuition and invention, is to prepare the accord of intelligences to render them tolerant and conciliatory.

\section{W. D. BATEN}

Science in a Tavern. By Charles S. Slichter. Madison, Wisconsin, University Press, 1938. $9+186 \mathrm{pp}$.

The title of this book refers to the meetings in London taverns and private houses, beginning about 1650, of English scientists and men of means and philosophical interests. At these meetings a great deal was eaten and drunk, discussion and even experimentation took place. It was from this nucleus that the Royal Society was formed in 1662. After that an inner circle still dined before the meetings of the Society and in 1725 formed a dining club. Dean Slichter, in the first two essays of this volume sketches the parallel early histories of the Royal Society and of the Club, stressing the importance for science of the patronage of the men of wealth and social position who belonged to these organizations. 